\title{
ASSOCIAÇÃO ENTRE A INCIDÊNCIA DO LEVANTAMENTO DE ÍNDICE RÁPIDO DE AEDES AEGYPTI (LIRAA) E AS CONDIÇÕES CLIMÁTICAS EM UBERLÂNDIA, MINAS GERAIS, BRASIL, ENTRE 2014 A 2016
}

\author{
Elisângela de Azevedo Silva Rodrigues \\ Doutora em Geografia, Laboratório de Geografia Médica e Vigilância em Saúde do Núcleo de Educação em \\ Saúde Ambiental (NESA) \\ Universidade Federal de Uberlândia - UFU \\ elisangelarodrigues@yahoo.com.br \\ Samuel do Carmo Lima \\ Doutor em Geografia, Professor do Instituto de Geografia \\ Universidade Federal de Uberlândia - UFU \\ samuel@ufu.br
}

\begin{abstract}
RESUMO
O presente trabalho foi desenvolvido com o objetivo de correlacionar os dados do Levantamento de Índice Rápido para Aedes aegypti (LIRAa) com as condições climáticas, no período de 2014 a 2016, no município de Uberlândia, Minas Gerais. A pesquisa foi realizada por meio do levantamento de dados do LIRAa, através do banco de dados da Secretaria Municipal de Saúde (SMS) e do Laboratório de Clima e Recursos Hídricos (LCRH) da Universidade Federal de Uberlândia (UFU). Foram ainda realizados testes estatísticos, utilizou-se o programa Bioestat 5.0. A regressão Linear Múltipla foi utilizada para analisar a variação dos dados meteorológicos: temperatura, precipitação pluviométrica, umidade relativa do ar em relação aos LIRAa's de 2014 a 2016. Os resultados foram considerados insignificantes, tendo por parâmetro a probabilidade ( $p$ ) do erro foi inferior a $5 \%$ ( $p$ $<0,05)$.
\end{abstract}

Palavras-chave: Levantamento Rápido de Índices para Aedes Aegypti (LIRAa). Aedes aegypti. Dengue.

\section{ASSOCIATION BETWEEN THE IMPACT OF AEDES AEGYPTI (LIRAA) FAST INDEX SURVEY AND CLIMATE CONDITIONS IN UBERLAND, MINAS GERAIS, BRAZIL, BETWEEN 2014 TO 2016}

\begin{abstract}
The present work was developed with the objective of correlating the data of the Fast Index Survey for Aedes aegypti (LIRAa) with the climatic conditions, in the period from 2014 to 2016, in the city of Uberlândia, Minas Gerais. The research was carried out through the data collection of the LIRAa, through the database of the Municipal Health Secretariat (SMS) and the Laboratory of Climate and Water Resources (LCRH) of the Federal University of Uberlândia (UFU). Statistical tests were also performed, using the program Bioestat 5.0. The Multiple Linear regression was used to analyze the variation of the meteorological data: temperature, rainfall, relative air humidity in relation to LIRAa's from 2014 to 2016. The results were considered insignificant, taking as parameter the probability $(p)$ of the error was lower to $5 \%(p<0.05)$.
\end{abstract}

Keywords: Rapid Survey of Indexes for Aedes Aegypti (LIRAa). Aedes aegypti. Dengue. 


\section{INTRODUÇÃO}

A Dengue é uma doença febril aguda, nos últimos anos, tem se tornado crescente problema de saúde pública, sendo uma epidemia de ameaça global. A doença é causada por quatro sorotipos do vírus: DENV-1, DENV-2, DENV-3 e DENV-4. A infecção por um deles confere proteção permanente para o mesmo sorotipo e imunidade parcial e temporária contra os outros três tipos (BRASIL, 2001; 2010; COSTA \& FORBELONI, 2019.

Segundo a Organização Mundial de Saúde (OMS), aproximadamente dois bilhões e meio de pessoas vivem em áreas de risco de transmissão de Dengue e a doença é endêmica em mais de 100 países de todos os Continentes, com exceção da Europa. A OMS estima que, no mundo, ocorram entre 50 e 100 milhões de casos, resultando em cerca de 500 mil internações e 20 mil óbitos por ano. Segundo - Ministério de Saúde o número de casos de dengue triplicou, no período de 2006 a 2010, aumentando os registros de 345.992 casos para um milhão de casos (SINAN, 2018).

Até os dias atuais, vários métodos para aferir os níveis de infestação do Aedes aegypti e indicadores de risco da transmissão de Dengue têm sido empregados.Os indicadores vetoriais servem para o monitoramento de risco de ocorrência dos casos de Dengue (MARANGONI FILHO, 2012).

O Levantamento de Índice Rápido para Aedes aegypti (LIRAa é um método amostral desenvolvido pelo Programa Nacional de Controle de Dengue (PNCD-MS), lançado em 2003, que monitora a densidade larvária a partir dos indicadores: Índice Predial (IP) e Índice de Breateau (IB). Ele tem sido utilizado para substituir o levantamento de índice tradicional, que, normalmente, apresenta o resultado somente após o fechamento do ciclo bimestral do trabalho. $O$ LIRAa) representa um método simplificado aleatório de determinação de dados sobre a infestação do vetor Aedes aegypti, possibilitando a obtenção de informações para direcionamento do trabalho de rotina em campo e interferência emergencial nos espaços intra-urbanos com maior densidade larvária do Município, contribuindo assim, para estruturar ações específicas e maior efetividade no combate vetorial (BRASIL, 2013; UBERLANDIA, 2015). O LIRAa corresponde a um levantamento de índice larvários do Aedes aegypti que utiliza como unidade de análise aglomerados intra-urbanos denominados estratos, com a hipótese de que possa refletir a situação epidemiológica do município, funcionando como preditor do risco da Dengue (BRASIL, 2013).

O presente trabalho teve por objetivo de correlacionar o Levantamento de Índice Rápido (LIRAa) com as condições climáticas, no período de 2014 a 2016, no município de Uberlândia, Minas Gerais.

\section{MATERIAL E MÉTODOS}

O município de Uberlândia está localizado na porção sudoeste do Estado de Minas Gerais, região do

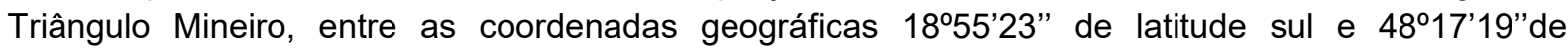
longitude oeste, a uma altitude média 863 metros. Ocupa uma área total de $4.115,09 \mathrm{~km}^{2}$, sendo que $219,00 \mathrm{~km}^{2}$ são ocupados pela zona urbana e $3.896,822 \mathrm{~km}^{2}$, pela zona rural (IBGE, 2010) (Figura 1).

O clima é tropical semiúmido com verão chuvoso e inverno seco. A precipitação média anual é de 1500-1600 mm, com forte concentração de chuvas nos meses de dezembro a fevereiro. A temperatura média mensal varia de $20,9^{\circ} \mathrm{C}$ a $23,1^{\circ} \mathrm{C}$ e o período mais quente do ano se estendem de outubro a abril. A vegetação é típica de cerrado e a hidrografia bastante rica. A população de Uberlândia foi estimada, em 2013, em 646.673 habitantes e constitui-se num importante entroncamento rodoferroviário, que facilita a comunicação com os principais centros urbanos das regiões Sudeste e Centro-Oeste. 
Figura 1 - Mapa de localização do município de Uberlândia, Minas Gerais, 2012.

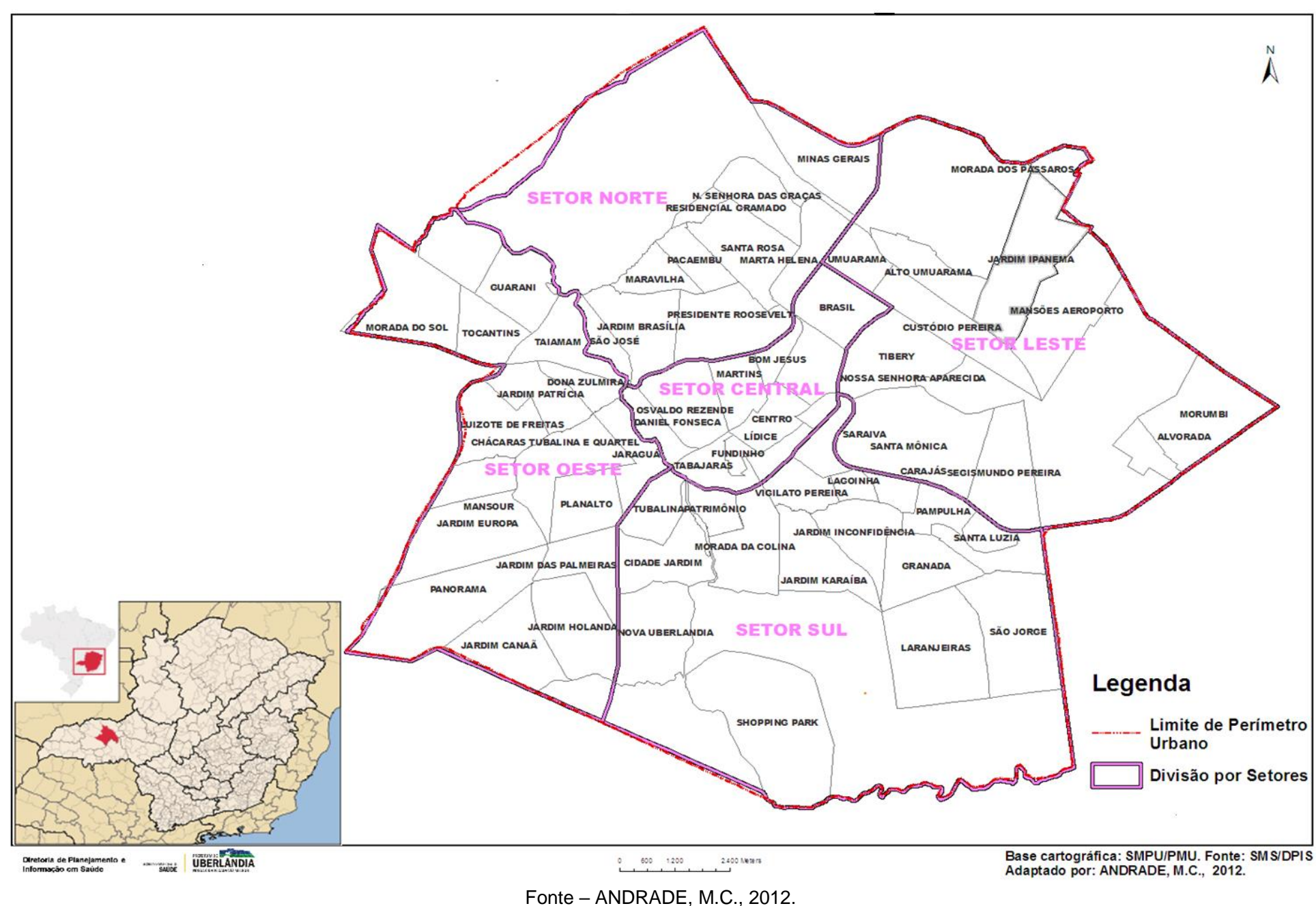


O município de Uberlândia está dividido em cinco Setores político-administrativos: Setor Norte, Setor Sul, Setor Oeste, Setor Leste e Setor Central (IBGE, 2010; 2013).

Para a realização desta pesquisa os dados foram fornecidos pelo Centro de Controle de Zoonoses de Úberlândia. A primeira etapa do LIRAa foi o reconhecimento geográfico. Para o reconhecimento geográfico, foram utilizados mapas (croquis) com a delimitação dos bairros, a informação sobre o número dos quarteirões e dos imóveis.

No LIRAa, os quarteirões de todos os setores foram sorteados, de acordo com os 27 estratos designados no Município de Uberlândia, de modo que, foram estimados o IP e o IB. O IP é utilizado para obter o percentual de casas positivas com a presença de Aedes aegypti. O IB é comumente utilizado para fazer a relação entre o número de recipientes positivos e o número de imóveis pesquisados.

\begin{tabular}{|c|c|}
\hline & Índice Predial \\
\hline \multirow[t]{2}{*}{$\mathrm{IP}=$} & $\mathrm{N}^{\circ}$ imóveis positivos \\
\hline & $\mathrm{N}^{\circ}$ imóveis pesquisados \\
\hline \multirow{3}{*}{$\mathrm{IB}=$} & Índice Breteau \\
\hline & Recipientes positivos \\
\hline & $\mathrm{N}^{\circ}$ imóveis pesquisados \\
\hline
\end{tabular}

Os recipientes/depósitos considerados criadouros do Aedes aegypti que continham água foram classificados em 5 (cinco) grupos: A,B,C,D,E, de acordo com o quadro 1:

\section{Quadro 1 - Classificação de criadouros do Aedes aegypti.}

\begin{tabular}{|c|l|}
\hline A1 & $\begin{array}{l}\text { Depósito d'água elevado ligado à rede pública e/ou sistema de captação mecânica em poço, } \\
\text { cisterna ou mina d'água: caixas de água, tambores, depósitos de alvenaria. }\end{array}$ \\
\hline A2 & $\begin{array}{l}\text { Depósitos ao nível do solo para armazenamento doméstico: tonel, tambor, barril, tina, depósitos } \\
\text { de barro (filtros, moringas, potes), cisternas, caixa de água, captação de água em } \\
\text { poço/cacimba/cisterna. }\end{array}$ \\
\hline B & $\begin{array}{l}\text { Vasos/frascos com água, prato, garrafas, pingadeiras, recipientes de degelo em geladeiras, } \\
\text { bebedouros em geral, pequenas fontes ornamentais, materiais em depósitos de construção } \\
\text { (sanitários estocados, etc.), objetos religiosos / rituais. }\end{array}$ \\
\hline C & $\begin{array}{l}\text { Tanques em obras, borracharias e hortas, calhas, lajes e toldos em desníveis, ralos, sanitários } \\
\text { em desuso, piscinas não tratadas, fontes ornamentais; floreiras/vasos em cemitérios; cacos de } \\
\text { vidro em muros, outras obras arquitetônicas (caixas de inspeção/passagens). }\end{array}$ \\
\hline D1 & Pneus e outros materiais rodantes (câmaras-de-ar, manchões). \\
\hline D2 & $\begin{array}{l}\text { Lixo (recipientes plásticos, garrafas, latas); sucatas em pátios e ferros velhos (PE), entulhos de } \\
\text { construção. }\end{array}$ \\
\hline E & $\begin{array}{l}\text { Axilas de folhas (bromélias, etc.), buracos em árvores e em rochas, restos de animais (cascas, } \\
\text { carapaças, etc.). }\end{array}$ \\
\hline
\end{tabular}

Fonte - Manual LIRAa: Metodologia para avaliação dos Índices de Breteau, Predial e tipos de recipientes, 2013.

A inspeção do município foi realizada nos imóveis e nos terrenos baldios. Nos edifícios, foi inspecionado apenas o térreo conforme preconizado no Manual: Metodologia para avaliação dos Índices de Breteau, Predial e tipos de recipientes (BRASIL, 2013).

$\begin{array}{lllll}\text { Caminhos de Geografia } & \text { Uberlândia } & \text { v. 20, n. } 72 & \text { Dez/2019 } & \text { p. 251-263 Página } 254\end{array}$


Após o sorteio dos quarteirões, foram sorteadas as residências. A inspeção foi feita em $20 \%$ das residências, nos depósitos onde continham água. A pesquisa foi iniciada no primeiro imóvel do quarteirão e, com deslocamento no sentido horário, contam-se quatro imóveis, para a seguir inspecionar-se o sexto imóvel ( $2^{\underline{a}}$ amostra), e assim sucessivamente, inspecionou-se um em cada cinco imóveis. No caso de imóveis fechados ou recusa da inspeção, o Agente de Controle de Zoonoses fazia a substituição do imóvel pelo anterior e, caso ocorresse do anterior fechado ou recusado, era feita naquele imediatamente posterior (BRASIL, 2013) (Figura 2).

Figura 2 - Sequência de inspeção no quarteirão sorteado.

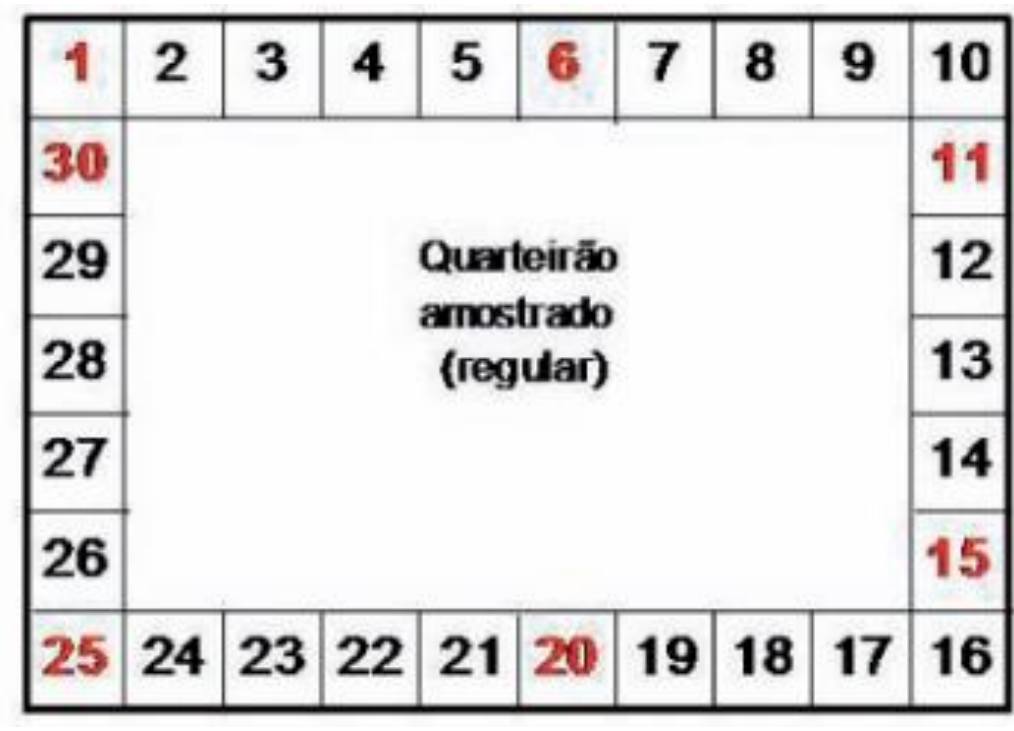

Fonte - Manual LIRAa (2013).

Os Agentes de Controle de Zoonoses utilizaram pesca-larva para percorrer o depósito até o fundo, em forma de oito, logo após o material retido na pesca-larva foi transferido para uma pequena bacia com água limpa. Com auxílio de uma pipeta as larvas e pupas foram colocadas na palma da mão e virando em tubito com a preparação de álcool a setenta (70\%). Os tubitos foram rotulados com o número do quarteirão, endereço, quantidade de larvas e pupas e tipos de depósitos em que foram encontradas e em seguida as informações foram registradas em Boletim de Campo e Laboratório (BCL) (BRASIL, 2013).

Em seguida os tubitos foram levados para o laboratório de Entomologia do Centro de Controle de Zoonoses. No laboratório, as larvas foram colocadas na placa de petri e com o auxílio do pincel para virar as larvas. A identificação das larvas/pupas foi feita em lupa estereomicroscópica (BRASIL, 2013).

A etapa final consta da introdução dos dados no SINAN (Sistema de Agravos de Notificação) e no Programa Padrão para os municípios. Este último trata-se de um programa com planilhas eletrônicas onde são inseridos os dados das amostras dos Estratos, o qual faz o cálculo dos parâmetros estatísticos e do resultado do LIRAa.

Considerou-se na presente pesquisa que valores abaixo de $1 \%$ configuram IIP baixo, entre $1,1 \%$ a $3,9 \%$ IIP médio e acima de $4 \%$ IIP alto de acordo com os critérios pré-estabelecidos pelo Ministério da Saúde (BRASIL, 2013). 
Os dados abióticos/climatológicos (temperatura, precipitação pluviométrica, umidade relativa) da pesquisa foram obtidos no Laboratório de Climatologia e Recursos Hídricos (LCRH) da UFU.

Foi realizado o teste estatístico utilizando-se do programa Bioestat 5.0. A Regressão Linear Múltipla foi utilizada para analisar a variação dos dados metereológicos: temperatura e umidade relativa.

Para confecção dos mapas foram utilizados o programa QGIS 2.6.1 Brighton e o estimador Kernel Quártico (SILVA et.al, 2014).

\section{RESULTADOS E DISCUSSÕES}

O PNCD preconiza que os índices de infestação predial (IIP) inferior a 1\% são considerados em condições satisfatórias, teoricamente, sem risco de transmissão de doenças pelo Aedes aegypti. A tabela 1 se refere à quantidade de bairros com IIP baixo, médio e alto, casos de Dengue por trimestre e óbitos, no período de 2014 a 2016.

Foram realizadas 8 LIRAa's em Uberlândia entre os anos de 2014 a 2016. Em média, 31 bairros (42\%) estiveram com índices de IIP baixos, 26 bairros (35\%) estiveram com IIP médio e $17(23 \%)$ estiveram com IIP alto (Tabela 1$)$.

Tabela 1 - Índice de Infestação Predial, casos de dengue e óbitos por bairro no município de Uberlândia, no período de 2014 a 2016.

\begin{tabular}{|c|c|c|c|c|c|}
\hline LIRAa & \multicolumn{3}{|c|}{ QT.BAIRROS } & \multirow{2}{*}{$\begin{array}{l}\text { Casos de } \\
\text { Dengue* }\end{array}$} & \multirow{2}{*}{ ÓBITOS } \\
\hline & IIP BAIXO & IIP MÉDIO & IIP ALTO & & \\
\hline 1\%/2014 & 15 & 30 & 29 & 1.564 & 0 \\
\hline 2\%/2014 & 22 & 22 & 30 & 2.484 & 3 \\
\hline 3\%/2014 & 39 & 33 & 2 & 202 & 0 \\
\hline $1 \% / 2015$ & 22 & 29 & 23 & 3.626 & 2 \\
\hline 2\%/2015 & 14 & 17 & 43 & 12.260 & 7 \\
\hline 3\%/2015 & 45 & 26 & 3 & 510 & 0 \\
\hline 1\%/2016 & 54 & 18 & 2 & 3.873 & 0 \\
\hline $2^{\circ} / 2016$ & 35 & 34 & 05 & 5.163 & 2 \\
\hline Média & $31(42 \%)$ & $26(35 \%)$ & $17(23 \%)$ & 3710 & 2 \\
\hline
\end{tabular}

Fonte - SMS/ CCZ-UDI/VIGEP, 2016; casos de dengue no Trimestre.

Houve a predominância dos bairros com IIP alto e médio nos setores Sul e Oeste, IIP baixos no setor Leste e IIP médios e baixos nos setores Central e Norte.

Percebe-se que a maior quantidade de bairros com IIP alto coincidem com $02^{\circ}$ LIRAa independente do ano e o maior o número de casos de dengue e maior número de óbitos por dengue compreende o $2^{\circ}$ trimestre (abril a junho). Também o IIP é sempre maior no segundo LIRAa (março e abril), sendo o maior índice verificado em 2015 (6,2\%) e no 3o LIRAa, realizado em outubro é sempre menor.

Curiosamente, o menor LIRAa registrado no período foi justamente no $3^{\circ}$ LIRAa de 2015 (outubro), talvez como reação do serviço de controle vetorial e mobilização social, tendo em vista o agravamento da situação epidemiológica da transmissão da dengue no Município do início do ano (Gráfico 1). 
Gráfico 1 - Índice de infestação de Aedes aegypti em Uberlândia, 2014 a 2016.

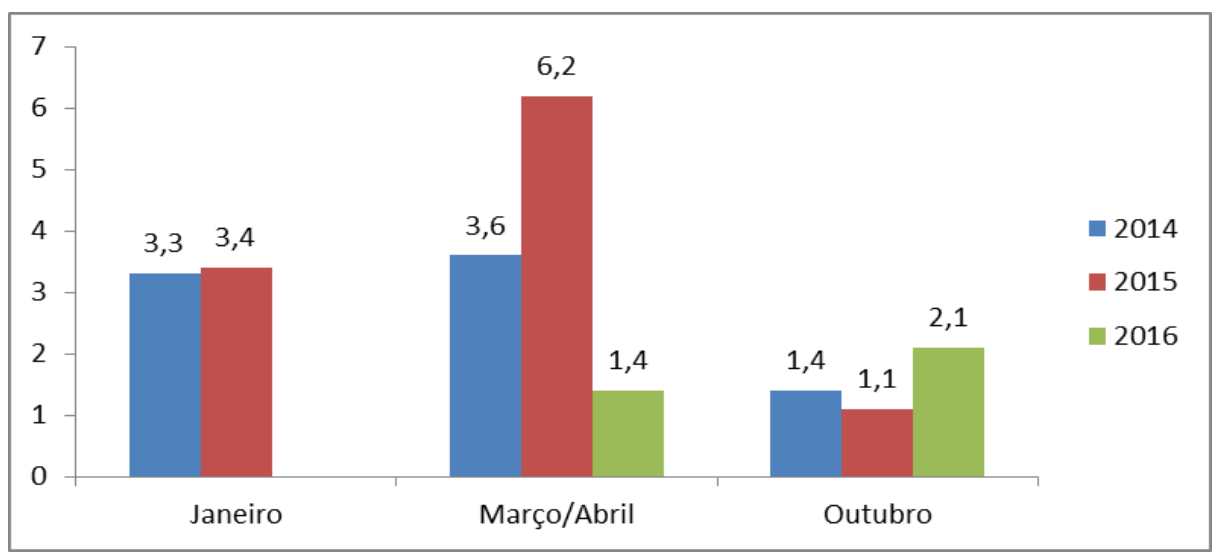

Fonte - SMS, CCZ- Uberlândia, 2014-2016.

No ano de 2014 foram realizados três LIRAa's, sendo inspecionadas 33.612 imóveis.

No $1^{\circ}$ LIRAa de 2014 (janeiro), o número de imóveis urbanos estimados era de 268.537, o número de imóveis programados para pesquisa foi de 11.431 e o número de imóveis inspecionados foi 11.008. O Índice predial foi 3,3\% e o Índice Breteau, 4,0\%.

No $2^{\circ}$ LIRAa de 2014 (março), o número de imóveis urbanos estimados e o número de imóveis pesquisados foram os mesmos do LIRAa de janeiro- O Índice predial foi 3,3\% e o Índice Breteau, $4,0 \%$.

No 3ㄹ LIRAa de 2014 (outubro), o número de imóveis urbanos estimados era de 274.408 , o número de imóveis programados para pesquisa foi de 11.625 e o número de imóveis inspecionados foi 11.389 . O Índice predial foi $1,4 \%$ e o Índice Breteau, 1,7\%. Somente dois $(2,7 \%)$ bairros apresentaram alto risco, 33 bairros $(44,6 \%)$ apresentam médio risco e 39 bairros $(52,7 \%)$ em baixo risco para transmissão de doenças pelo mosquito.

No ano de 2015 foram realizados três LIRAa's, sendo inspecionados 33.057 imóveis. No $1^{\circ}$ LIRAa de 2015 (janeiro), o número de imóveis urbanos estimados era de 268.537, o número de imóveis programados para pesquisa foi de 11.625 e o número de imóveis inspecionados foi 11.107. O Índice predial foi 3,4\% e o Índice Breteau, 4,3\%.

No 2ํㄴ LIRAa de 2015 (março), o número de imóveis urbanos estimados era de 273.656, o número de imóveis programados para pesquisa foi de 11.629 e o número de imóveis inspecionados foi 11.629. O Índice predial foi 6,2\% e o Índice Breteau 9,3\%.

A pior situação epidemiológica foi verificada no $2^{\circ}$ LIRAa de 2015, quando 43 dos 74 bairros de Uberlândia estiveram em IIP alto, tendo sido registrado 12.260 casos de dengue e 7 óbitos no trimestre (janeiro, fevereiro e março).

A maior quantidade de bairros com IIP alto coincidem com o $2^{\circ}$ LIRAa independente do ano e 0 maior o número de casos de dengue e maior número de óbitos por dengue compreende o $2^{\circ}$ trimestre (abril a junho). Também o IIP é sempre maior no segundo LIRAa (março e abril), sendo o maior índice verificado em $2015(6,2 \%)$ e no $3^{\circ}$ LIRAa, realizado em outubro é sempre menor. Curiosamente, o menor LIRAa registrado no período foi no $3^{\circ}$ LIRAa de 2015 (outubro), talvez como resultado do serviço de controle vetorial e a mobilização social, tendo em vista o agravamento da situação epidemiológica da transmissão da dengue no Município do início do ano.

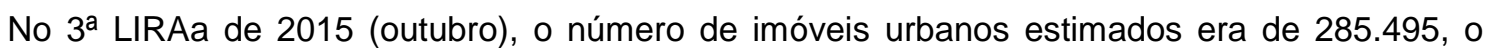
número de imóveis programados para pesquisa foi de 11.629 e o número de imóveis inspecionados foi 10.795. O Índice predial foi 1,1\% e o Índice Breteau, 1,3\%.

No ano de 2016 foram realizados apenas 2 LIRAa's, em abril e outubro. O LIRAa de janeiro foi suprimido devido o estado de calamidade em saúde pública decretado pelo governo federal, indicando a necessidade de intensificar no início do ano o trabalho de vistoria dos imóveis e eliminação dos criadouros do mosquito. Nestes 2 LIRAa's foram inspecionados 22.264 imóveis.

\begin{tabular}{llllll}
\hline Caminhos de Geografia & Uberlândia & v. 20, n. 72 & Dez/2019 & p. 251-263 Página 257
\end{tabular}


Figura 4 - Mapa do Índice de infestação predial de Aedes aegypti em Uberlândia, 는 LIRAa/março de 2015.

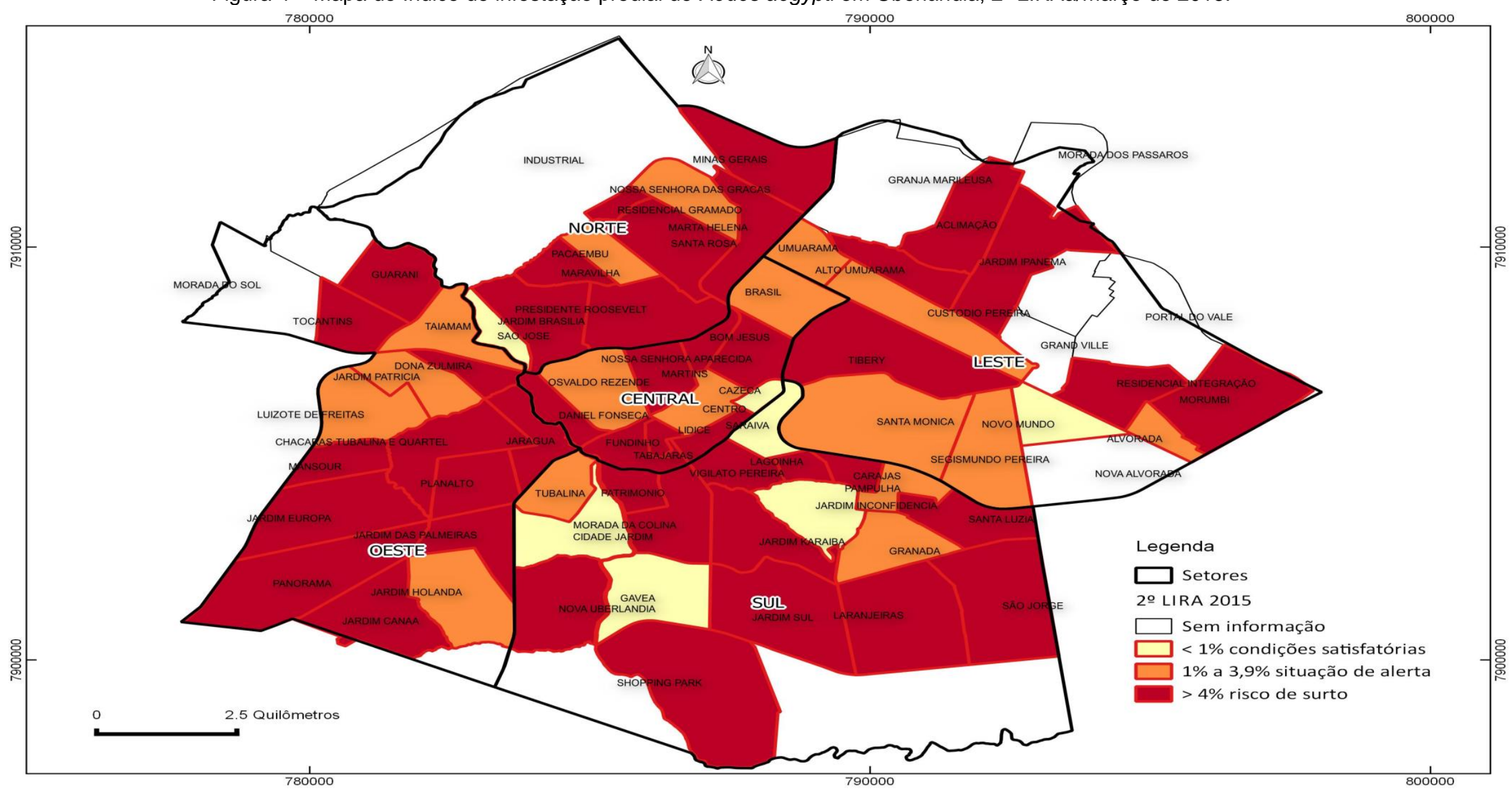

Fonte - SMS/CCZ (2015). Elaborado por: COSTA, I.M. 
No $1^{\circ}$ LIRAa de 2016 (abril), o número de imóveis urbanos estimados era de 306.756, o número de imóveis programados para pesquisa foi de 11.652 e o número de imóveis inspecionados foi 10.956. O Índice predial foi 1,4\% e o Índice Breteau, 1,6\%.

No $2^{\circ}$ LIRAa de 2016 (outubro), o número de imóveis urbanos estimados foi de 306.756, o número de imóveis programados para pesquisa foi de 11.653 o número de imóveis inspecionados foi 11.308. O Índice predial foi 2,1\% e o Índice Breteau 2,8\%.

Os indicadores vetoriais servem para a identificação dos criadouros do mosquito predominantes e são utilizados para monitoramento do risco de ocorrência de doenças transmitidas pelo Aedes aegypti. Marangoni Filho (2012), estudando os indicadores do LIRAa e ocorrência de Dengue, em Penápolis (SP), no período de 2006-2010, concluiu que a eficácia das atividades de controle será maior se incrementadas imediatamente após a aferição, no período mínimo de 2 (dois) meses após o LIRAa.

Para o direcionamento das ações de controle é de suma importância o conhecimento de fatores que influenciam a densidade populacional dos vetores tais como os fatores climáticos, a estrutura urbana de saneamento e os aspectos socioeconômicos BHATT et al., 2013). Na tabela 2, dos vários bairros que apresentaram o IIP alto, alguns, tiveram maior número de casos de Dengue, sendo reincidentes nos anos de 2014 a 2016. No Setor Leste estes bairros são Morumbi, Santa Mônica e Tibery; no Setor Oeste: Luizote de Freitas; no Setor Norte: Presidente Roosevelt e Jardim Brasília; no Setor Sul: São Jorge e Laranjeiras e no Setor Central: Martins e Brasil.

Tabela 2 - Bairros com maior número de casos de Dengue, no município de Uberlândia, no período de2014 a 2016.

\begin{tabular}{l|l|c|c|c|c|c|c}
\hline Setor & Bairro & $\mathbf{2 0 1 4}$ & $\%$ & $\mathbf{2 0 1 5}$ & $\%$ & $\mathbf{2 0 1 6}$ & $\%$ \\
\hline \multirow{4}{*}{ Leste } & lorumbi & 115 & 2,6 & 1006 & 6,0 & 464 & 4,9 \\
\cline { 2 - 7 } & Santa Mônica & 320 & 7,3 & 870 & 5,2 & 503 & 5,3 \\
\cline { 2 - 7 } & Tibery & 203 & 4,6 & 592 & 3,5 & 315 & 3,3 \\
\hline \multirow{2}{*}{ Oeste } & Luizote de Freitas & 193 & 4,4 & 814 & 4,9 & 289 & 3,1 \\
\hline \multirow{2}{*}{ Sult } & Pres. Roosevelt & 136 & 3,1 & 554 & 3,3 & 652 & 6,9 \\
\cline { 2 - 8 } & Jd. Brasília & 150 & 3,4 & 466 & 2,8 & 542 & 5,7 \\
\hline \multirow{2}{*}{ Central } & São Jorge & 376 & 8,5 & 915 & 5,5 & 306 & 3,2 \\
\cline { 2 - 8 } & Laranjeiras & 108 & 2,4 & 236 & 1,4 & 111 & 1,2 \\
\cline { 2 - 8 } & Martins & 98 & 2,2 & 580 & 3,5 & 358 & 3,8 \\
\hline
\end{tabular}

Fonte - SINAN/VIGEP, 2017.

Ao analisar a distribuição espacial e temporal da infestação do Aedes aegypti, percebeu-se que os índices são mais elevados na estação do Verão, geralmente quando ocorrem a pesquisa do $1^{\circ}$ LIRAa e $2^{\circ}$ LIRAa, realizados nos meses de janeiro e março, respectivamente. Este período é compreendido por maior pluviosidade, reduzindo-se o índice a partir do inverno. Contrariando a lógica homogênea dos maiores índices serem somente no $1^{\circ}$ e $2^{\circ}$ LIRAa, foi identificada uma situação diferente. Em alguns bairros também foram constatados IIP alto apesar do período seco no LIRAa realizado em outubro.

Em 2014, no 1ำ LIRAa, 94\% dos recipientes positivos estavam nas residências e $6 \%$ nos terrenos baldios. Nas residências, $9 \%$ dos focos estavam no intradomicílio e $91 \%$ no peridomicílio, ou seja, na área externa das residências como os quintais e varandas. No $2^{\circ}$ LIRAa de 2014, 93\% dos focos estavam nas residências e 7\% nos terrenos baldios. Nas residências, $4 \%$ dos focos estavam no intradomicílio e $96 \%$ no peridomicílio. E no 30 LIRAa de $2014,100 \%$ dos focos foram encontrados nas residências, sendo $16 \%$ no intradomicílio e $84 \%$ no peridomicílio. Neste último LIRAa, houve um aumento do percentual de focos encontrados no intradomicílio. Neste caso, a eliminação dos focos do Aedes aegypti por parte dos Agentes de Controle de Zoonoses fica prejudicado, dependendo mais diretamente da ação dos

Caminhos de Geografia Uberlândia $\quad$ v. 20, n. $72 \quad$ Dez/2019 $\quad$ p. 251-263 Página 259


moradores. Em 2015, no 1 LIRAa, 94\% dos recipientes positivos estavam nas residências e $6 \%$ nos terrenos baldios. Dos focos nos imóveis, $7 \%$ estavam no intradomicílio e $93 \%$ no peridomicílio. No 2 ㄴ LIRAa de 2015, 90,5\% dos focos estavam nos imóveis e 9,5\% em terrenos baldios. No domicílio, os $7 \%$ dos focos estavam no intradomicílio e $93 \%$ no peridomicílio. No 3 o LIRAa de 2015, $97,5 \%$ dos focos foram encontrados nas residências e $2,5 \%$ nos terrenos baldios, $10 \%$ no intradomicílio e $90 \%$ no peridomicílio. Novamente neste $3^{\circ}$ LIRAa o percentual de focos intradomiciliares aumentaram.

Percebeu-se que no $3^{\circ}$ LIRAa dos anos de 2014 e 2015 o aumento dos percentuais de focos do Aedes aegypti encontrados no intradomicílio. A infestação de uma casa é resultado das práticas de manejo de recipientes pelos seus ocupantes e da ecologia do comportamento de Aedes aegypti na postura de ovos. Durante o verão aumenta a incidência de chuvas e o Aedes aegypti busca o intradomicílio para fazer o repasto sanguíneo para maturação de seus ovos e para fazer a oviposição (BARBOSA et al., 2019).

Em 2016, no 1ํ LIRAa, 95\% dos recipientes positivos estavam nas residências e $5 \%$ nos terrenos baldios. No $2^{\circ}$ LIRAa, $96 \%$ dos focos estavam nos imóveis e $4 \%$ em terrenos baldios. Não foram discriminados os percentuais encontrados no intradomicílio e no peridomicílio. Durante os LIRAa's de 2014, 2015 e 2016, o maior percentual dos focos de Aedes aegypti foram encontrados em depósito do tipo B, seguido pelos depósitos do tipo C, D1 e D2. Os depósitos do tipo B em 2014 eram 54,3\%, em 2015 eram 24,6\% e em 2016 eram 23,8\%. Os depósitos do tipo C eram 29,5\%,25,6\% e 20,3\%, respectivamente nos anos 2014, 2015 e 2016. Os depósitos do tipo D1 em 2014 eram 8,0\%, em 2015 eram 8,3\% e em 2016 eram 6,0\%. Os depósitos do tipo D2 em 2014 eram 2\%, em 2015, 29,5\% e em 2016 eram $32,95 \%$. Os depósitos do tipo E nos anos 2014, 2015 e 2016, foram respectivamente, $0,2 \%, 0,8 \%$ e $0,82 \%$.

De acordo com a figura 5 , os tipos de criadouros positivos predominantes para Aedes aegypti nos LIRAa's de 2014 e 2015 foi do tipo B, com 54,7\% e 31,8\%, respectivamente. Em 2016, o tipo de depósito predominante foi o D2 com $32,9 \%$.

Figura 5 - Tipos de criadouros positivos para Aedes aegypti, no LIRAa no período de 2014 a 2016.

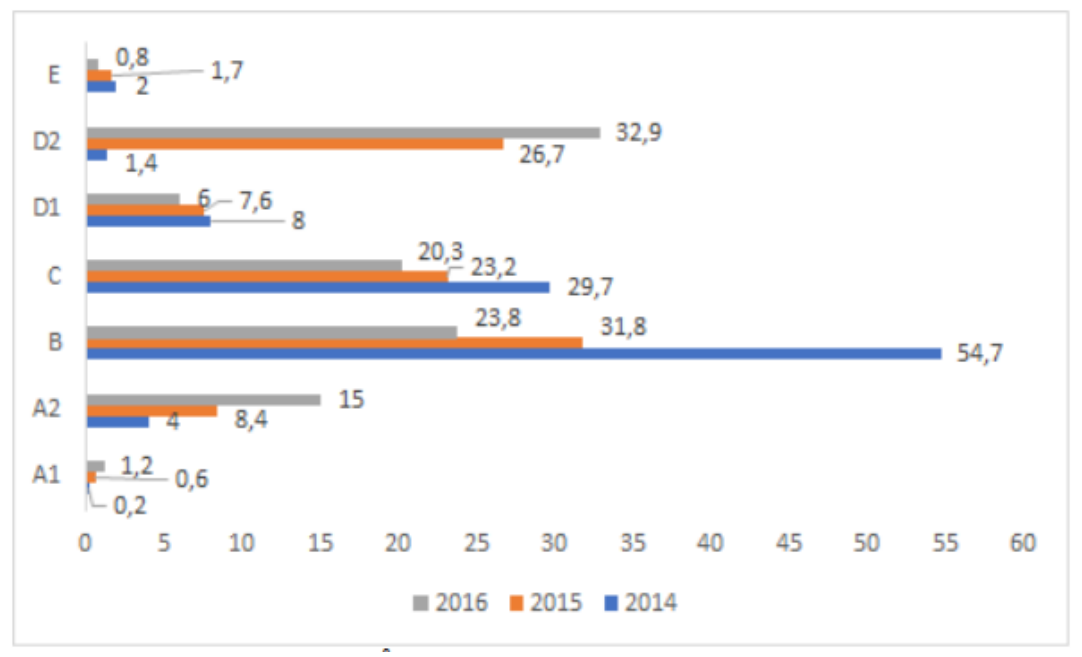

Com base nos tipos de focos encontrados em sua maioria do tipo B que são os vasos de plantas, frascos com água, prato, garrafas, pingadeiras, recipientes de degelo em geladeiras, bebedouros em geral, pequenas fontes ornamentais, materiais em depósitos de construção (sanitários estocados, etc.), objetos religiosos / rituais e os criadouros do tipo D2 composto por recipientes plásticos, garrafas, latas; sucatas em pátios, entulhos de construção. Verificou-se

Caminhos de Geografia Uberlândia $\quad$ v. 20, n. $72 \quad$ Dez/2019 $\quad$ p. 251-263 Página 260


que é preciso elaborar estratégias de ações educativas para a conscientização/sensibilização dos moradores no sentido de que haja mudança de hábitos a fim de evitar a produção de focos e consequentemente a proliferação do Aedes aegypti.

O índice de infestação predial de Aedes aegypti mostrou uma grande variação entre os meses, no período de 2014 a 2016, conforme os períodos sazonais de seca e de chuva. Constataramse associação diretamente proporcional entre o IIP e a pluviosidade.

De acordo com a Figura 6 a relação do IIP foi diretamente proporcional com médias mensais de precipitação $(\mathrm{mm})$, no período de 2014 e 2016, na área urbana de Uberlândia, Minas Gerais (Figura 6).

Figura 6 - IIP anual de Aedes aegypti e médias mensais de precipitação (mm), em Uberlândia, 2014 a 2016.

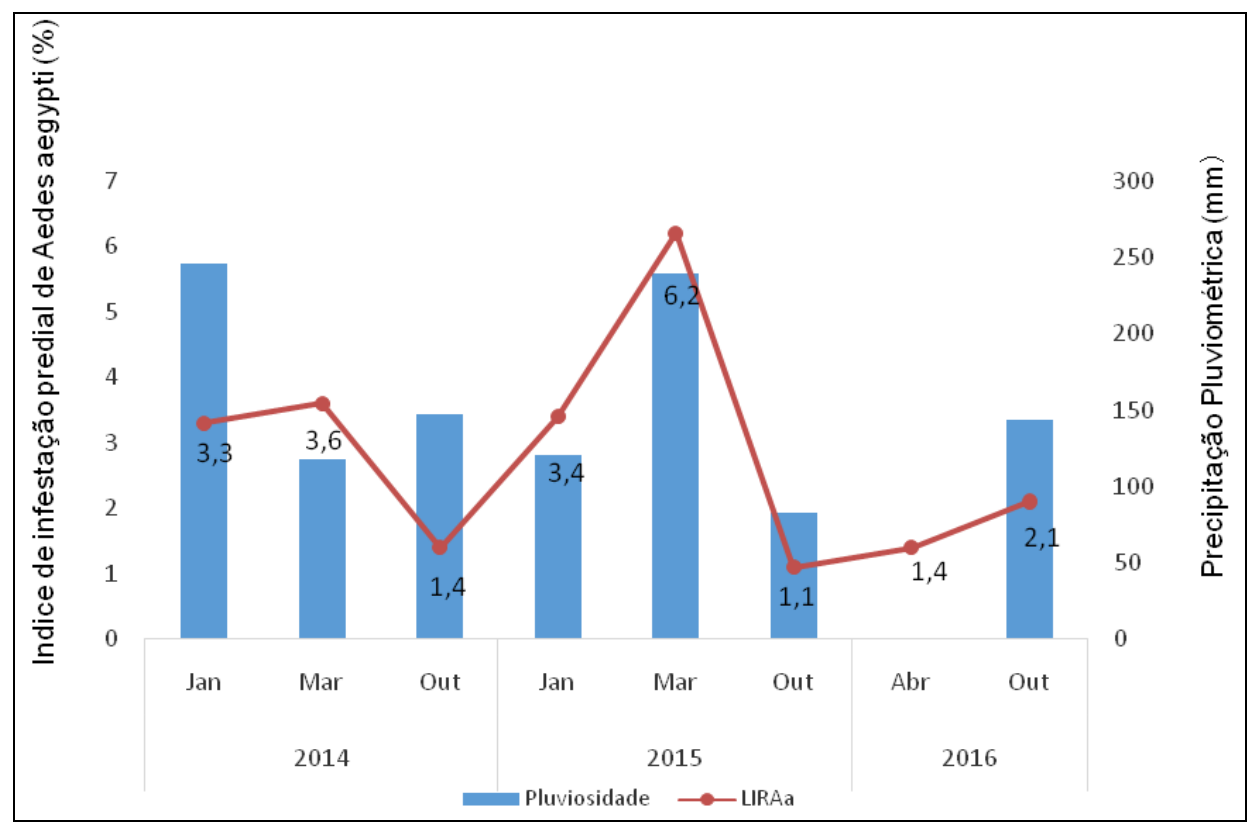

A regressão linear múltipla das variáveis de temperatura e pluviosidade teve como resultados: 0,04526 e 0,096473, em 2014; 0,084528 e 0,051046, em 2015 e; 0,092705 e -0,10767, em 2016. Em relação aos LIRAa's de 2014 a 2016 os resultados foram considerados insignificantes, tendo por parâmetro a probabilidade $(p)$ do erro foi inferior a $5 \%(p<0,05)$.

\section{CONSIDERAÇÕES FINAIS}

A análise dos dados do LIRAa demonstrou que alguns bairros como: Morumbi, Santa Mônica, São Jorge, Luizote de Freitas, Presidente Roosevelt e Brasil persistem sistematicamente em apresentar IIP alto nos três LIRAa's realizados durante o ano. Mesmo no $3^{\circ}$ LIRAa realizado em período seco estes bairros coincidiram com os bairros com maior número de casos notificados de Dengue no ano seguinte à pesquisa.

Os LIRAa's demonstraram também que os maiores índices de infestação não estão isolados por determinação social. Pelo contrário, foram tidos IIP alto em bairros de classe socioeconômica média também, principalmente devido ao cultivo de plantas ornamentais e vasos de planta com o pratinho que acumula água.

Apesar dos esforços apresentados pelo Programa de Controle de Dengue do município ainda está engessado no modelo utilizado desde o período das primeiras campanhas de Oswaldo

\begin{tabular}{lllll}
\hline Caminhos de Geografia $\quad$ Uberlândia & v. 20, n. 72 & Dez/2019 & p. 251-263 Página 261
\end{tabular}


Cruz. Pôde-se constatar que dentro dos índices de infestação apresentados, levanta questionamentos sobre a eficácia das estratégias de intervenções dos serviços de Vigilância em Saúde.

O modelo "campanhista" refere-se ao predomínio das intervenções públicas de saúde focalizadas em doenças endêmicas de natureza pontual (campanhas periódicas), centralizada em termos de concepção e controle da execução (na esfera federal), caracteriza-se ainda, principalmente nos primeiros meses do ano, na estação do Verão, há uma intensidade de informações comerciais veiculados pela mídia a respeito da doença, dos sintomas, das formas de prevenção do mosquito transmissor, como se tivesse apenas naquele período do ano e 0 resto do ano estivessem livres do Aedes.

A pesquisa apontou por meio do Índice de Breteau que a maioria dos focos do mosquito, sempre acima de $90 \%$ são encontrados na área peridomiciliar das residências. O índice de Breteau apontou que os criadouros como os pneus, as latas, os descartáveis jogados no quintal, o vaso de planta com pratinho, as vasilhas de água dos animais domésticos são os futuros possíveis focos para o mosquito e devem ser regularmente inspecionados, visto que os ovos do Aedes aegypti podem sobreviver sem contato com a água(KISTLER, 2015).Desta forma, é preciso resgatar que o Controle Vetorial seja de caráter permanente para que a população continue com as medidas de controle durante todo o ano.

É necessário buscar a articulação sistemática entre a vigilância epidemiológica e a vigilância entomológica com a atenção básica, integrando suas atividades de maneira a potencializar o trabalho considerando a participação de toda a sociedade.

As atividades de educação em saúde devem estar inseridas em todos os serviços que desenvolvam as ações de vigilância e controle do Aedes, requerendo o envolvimento efetivo das equipes multiprofissionais e multiinstitucionais com vistas ao trabalho articulado, nas diferentes unidades de prestação de serviços, divulgando sobre a ocorrência da doença, orientando a população para o reconhecimento de sinais clínicos da doença e, quando houver caso suspeito, a procura dos serviços de saúde para o diagnóstico precoce, o tratamento adequado e a notificação na Vigilância Epidemiológica.

\section{REFERÊNCIAS}

BHATT, S., Gething P.W., BRADY, O.J., MESSINA J.P., FARLOW, A.W., MOYES, C.L., DRAKE J.M., Brownstein J.S., HOEN A.G., SANKOH, O., MYERS, M.F., GEORGE, D.B., JAENISCH, T., WINT, G.R., SIMMONS, C.P., SCOTT, T.W., FARRAR, J.J., HAY, S.I. The global distribution and burden of dengue. Nature, v. 496, n. 7446, p. 504, 2013.

https://doi.org/10.1038/nature12060

BARBOSA, G. L., LAGE, M.O., ANDRADE, V.R., GOMES, A.H.A., QUINTANILHA, J.A., CHIARAVALLOTI-NETO, F. Influence of strategic points in the dispersion of Aedes aegypti in infested areas. Revista de saúde publica, v. 53, p. 29, 2019. https://doi.org/10.11606/S1518$\underline{8787.2019053000702}$

BRASIL. Dengue: instruções para pessoal de combate ao vetor: manual de normas técnicas. - 3. Ed., rev. - Brasília: Ministério da Saúde: Fundação Nacional de Saúde, 2001.84 p. : il. $30 \mathrm{~cm}$.

BRASIL, 2013. Ministério da Saúde. Secretaria de Vigilância em Saúde. Departamento de Vigilância das Doenças Transmissíveis. Levantamento Rápido de Índices para Aedes Aegypti (LIRAa) para vigilância entomológica do Aedes aegypti no Brasil: metodologia para avaliação dos índices de Breteau e Predial e tipo de recipientes / Ministério da Saúde, Secretaria de Vigilância em Saúde, Departamento de Vigilância das Doenças Transmissíveis - Brasília: Ministério da Saúde, 2013. $84 \mathrm{p}$.

CARVALHO, I.C.M. Educação ambiental: a formação do sujeito ecológico. Cortez, 2004. 
DEPRADINE, C.; LOVELL, E. Climatological variables and the incidence of Dengue fever in Barbados. International journal of environmental health research, v. 14, n. 6, p. 429-441, 2004. https://doi.org/10.1080/09603120400012868

DONALÍSIO, M. R.; GLASSER, C. M. Vigilância entomológica e controle de vetores do dengue. Revista Brasileira de Epidemiologia, v. 5, n. 3, p. 259-272, 2002. https://doi.org/10.1590/S1415-790X2002000300005

IBGE. Instituto Brasileiro de Geografia e Estatística. Sinopse do Censo Demográfico 2010. Brasília: Ministério do Planejamento e Orçamento, 2011. Disponível em <http://www.ibge.gov.br/home/estatistica/populacao/censo2010/primeiroos_resultados/populaca o_por_municipio.shtm> Acesso: junho de 2011.

IBGE. Instituto Brasileiro de Geografia e Estatística. Sinopse do Censo Demográfico 2010. Brasília: Ministério do Planejamento e Orçamento, 2011. Disponível em <http://www.ibge.gov.br/home/estatistica/populacao/censo2010/primeiroos_resultados/populaca o_por_municipio.shtm> Acesso: junho de 2011.

KISTLER, K. E.; VOSSHALL, L. B.; MATTHEWS, B. J. Genome engineering with CRISPRCas9 in the mosquito Aedes aegypti. Cell reports, v. 11, n. 1, p. 51-60, 2015. https://doi.org/10.1016/j.celrep.2015.03.009

MARANGONI FILHO, V. Indicadores entomológicos dos levantamentos rápidos de infestação de Aedes Aegypti e ocorrência de dengue na cidade de Penápolis, São Paulo-Brasil, 2006 a 2010, 41 p.. Dissertação (Mestrado em Medicina Veterinária) - Universidade Estadual Paulista, 2012.

PEREIRA, B.B.; W.E. Efeitos do butóxido de piperonila na toxicidade do organofosforado Temefós e o envolvimento de esterases na resistência de Aedes aegypti (Díptera: culicidae) ao Temefós. 2008.

SILVA, F. G.; MAGALHÃES, S. C. M. Correlação entre fatores climáticos, socioambientais e a dengue na microrregião Montes Claros/MG. Caminhos de Geografia, v. 18, n. 61, p. 231-244. 2017. https://doi.org/10.14393/RCG186115

UBERLÂNDIA. Áreas de abrangência. 2015. Disponível em: $<\mathrm{http}: / /$ www.uberlandia.mg.gov.br/uploads/cms_b_arquivos/13882.pdf>. Acesso em: 30 jul. 2016.

WORLD HEALTH ORGANIZATION. Dengue: guidelines for diagnosis, treatment, prevention and control, 2009.

Recebido em: 01/10/2018

Aceito para publicação em: 26/11/2019 